\title{
Anthracycline Chemotherapy in Treating Advanced Breast Cancer and its Effect on Estradiol and Tumor Size
}

\author{
HUIFEN ZHEN, G. FAN, Z. XIAOJUN, MIAOMIAO JIA, Y. HAIBO, Y. YARONG, L. YUANDONG AND JINNAN GAO* \\ Department of Breast Surgery, Shan Xi Bethune Hospital, Taiyuan ShanXi 030032, P. R. China
}

\section{Zhen et al.: Effects of Anthracycline Chemotherapy on Advanced Breast Cancer}

\begin{abstract}
To investigate the therapeutic effect of anthracycline chemotherapy in advanced breast cancer and its influence on estradiol and tumor size, 136 breast cancer patients in Shan Xi Bethune Hospital were divided into anthracycline chemotherapy group and non-anthracycline chemotherapy group. The clinical effects of anthracycline chemotherapy group and non-anthracycline chemotherapy group were observed. The levels of estrone, estradiol and follicle stimulating hormone before and after treatment were measured. The tumor size, adverse reactions and 2-y survival rate of anthracycline chemotherapy group and non-anthracycline chemotherapy group were measured after 1-3 courses of treatment. The total effective rate of anthracycline chemotherapy group and non-anthracycline chemotherapy group was 64.7 and $23.5 \%$, respectively and the total effective rate of non-anthracycline chemotherapy group was significantly lower than that of anthracycline chemotherapy group $(p<0.05)$. There was no significant difference in the comparison of serum estrone, estradiol and follicle stimulating hormone between the anthracycline chemotherapy group and non-anthracycline chemotherapy group $(\mathbf{p}<\mathbf{0 . 0 5})$. Compared to the anthracycline chemotherapy group, the level of serum estrone and estradiol in non-anthracycline chemotherapy group was significantly higher, compared to the non-anthracycline chemotherapy group, the level of serum follicle stimulating hormone in the anthracycline chemotherapy group was significantly higher $(\mathbf{p}<0.05)$. There was no significant difference in tumor size between the anthracycline chemotherapy group and non-anthracycline chemotherapy group $(p>0.05)$. After treatment, the tumor volume of each group gradually decreased with increased time of drug exposure and the degree of tumor reduction in the non-anthracycline chemotherapy group was lower than that of the anthracycline chemotherapy group $(p<0.05)$. Adverse reactions in both the groups were, the number of leucopenia cases in the anthracycline chemotherapy group was 14 while that in the non-anthracycline chemotherapy group was 17 . The number of adverse reactions in the non-anthracycline chemotherapy group was more than that in the anthracycline chemotherapy group, but the difference was not significant (p>0.05). After 2 y of followup, the survival rate of anthracycline chemotherapy group was significantly higher than that of nonanthracycline chemotherapy group $(p<0.05)$. The effect of anthracycline on advanced breast cancer was better, the level of serum estradiol decreased, and the volume of tumor decreased.
\end{abstract}

Key words: Breast cancer, anthracyclines, E2, clinical efficacy

Breast cancer is one of the common tumors in women, and it is also one of the reasons for the high mortality rate of women cancer patients. Recently significant progress has been made in treating breast cancer. Surgical resection is also an current effective treatment mode, which improved the quality of life of patients for 5 to $10 \mathrm{y}$. However, there is still metastasis and recurrence after radical mastectomy ${ }^{[1,2]}$. Early diagnosis and comprehensive treatment of breast cancer can effectively improve the 5-y and $10-y$ survival rate of patients. However, $30 \%$ of breast cancer patients still have the possibility of recurrence and metastasis, which causes many difficulties in clinical treatment ${ }^{[3]}$.
At present, anthracycline drugs such as daunorubicin, doxorubicin and Adriamycin have significant effect in clinical tumor management and are commonly used in clinical medicine. These drugs exert definite effect in the treatment of breast cancer, high mortality, high tolerance and low recurrence ${ }^{[4]}$. In this paper, epirubicin is selected as anthracycline drug. Epirubicin is one of the derivatives developed on the basis of anthracycline. Compared to its derivatives, epirubicin has a lower incidence of cardiotoxicity and its leakage effect is very good. It enters into cancer cell interior and controls disease progress by inhibiting nucleic acid generation and mitosis of cancer cells ${ }^{[5,6]}$. The

*Address for correspondence

E-mail: ftty1516@163.com 
growth and development of human breast tissue will be affected by many endocrine hormones, such as estrone (E1), estradiol (E2) and follicle stimulating hormone $(\mathrm{FSH})^{[7]}$. Estrogens are one of the important endocrine hormones of women, which play an important role in the diagnosis of breast cancer ${ }^{[8]}$. In this paper, the effect of anthracycline chemotherapy in the treatment of advanced breast cancer and effects on E2 levels and tumor size were studied.

One hundred and thirty six breast cancer patients including 2 males reported at the Shan Xi Bethune Hospital from March 2014 to July 2016 were included in this study. The age of the patients was $21 \mathrm{y}$ at the minimum and $74 \mathrm{y}$ at the maximum. According to the order of admission, the number of patients in the anthracycline chemotherapy $(\mathrm{NH})$ group and the same number were in the non-anthracycline chemotherapy group (conventional drugs treated, CG) with 68 cases in each group. The average age of patients in NH group was $45.23 \pm 3.03 \mathrm{y}$. The average age of patients in CG group was $45.31 \pm 2.87 \mathrm{y}$. There was no difference in the comparative clinical data between the $\mathrm{NH}$ group and CG group as shown in Table 1.

The inclusion criteria were, advanced breast cancer without metastasis, aged between 21-75 y, patients with complete admission and screening data and those in good physical condition during the study period. The exclusion criteria were those with abnormal function of other organs, those with multiple types of tumor at the same time, pregnant or lactating patients and those with poor communication ability.

Patients in the two groups were given nutrition support, dexamethasone and 5-HT3 receptor blocker before chemotherapy to prevent adverse reactions. In the NH group, patients were given epirubicin intravenously at a dose of $75 \mathrm{mg} / \mathrm{m}^{2}$ for one day and docetaxel was given the same dosage as epirubicin for one day, once in the course of treatment; in the CG group, capecitabine was given at a dose of $2 \mathrm{~g} / \mathrm{m}^{2}$, no more than $4 \mathrm{~g} / \mathrm{m}^{2}$ per day, for $2 \mathrm{w}$, during the course of treatment. The course of treatment was $21 \mathrm{~d}$ in the $\mathrm{NH}$ and the $\mathrm{CG}$ groups.
The clinical effects were observed in the $\mathrm{NH}$ and the $\mathrm{CG}$ groups. The levels of E1, E2 and FSH were monitored in both the NH and CG groups. The tumor size was monitored after 1-3 courses of treatment in both groups. Adverse reactions in both groups were monitored and compared. Survival rate in both the groups was monitored through follow-up for $2 \mathrm{y}$.

E1, E2 and FSH levels were determined by collecting $5 \mathrm{ml}$ of venous blood when the patients in the group were fasting once before and once after treatment. Plasma was separated by centrifugation and the changes in E1, E2 and FSH in plasma were measured using ELISA. Measurement of tumor size was using the select Siemens acusons 2000 ABVS (automated breast volume scanner) system, $1415 \mathrm{bv}$ high-frequency linear array probe the frequency of which was set to $11 \mathrm{MHz}$, scans with lateral, central and medial breast records were sent to the ABVS workstation, in which using the 3-D reconstruction method coronal and sagittal plane data was included, The largest section was selected to calculate the volume of the foci. Complete relief was considered when the pain on the affected side disappeared, the nipple had no beneficial fluid and when the lesions were absent. Partial remission was when more than $30 \%$ of lesion was found to shrink. Disease was considered as stable when shrinkage in the diameter and volume of the tumor could be observed, but not to achieve partial remission. Disease was considered to progress when more than $20 \%$ of the lesions increase in volume, or when new lesions appeared.

The changes in E1, E2 and FSH levels, survival rate and tumour volume before and after treatment were statistically analysed using GraphPad Prism 8 software. Chi square test was used for $n(\%)$, variable was used for and LSD t (least significant difference) or Bonferroni test was used for comparison between the 2 groups. Repeated measurement analysis of variance and log rank test were used for comparison between the 2 groups at different time points, with $\mathrm{p}<0.05$ as the difference remarkable.

The total effective rate of $\mathrm{NH}$ group and CG group was 64.7 and $23.5 \%$, respectively and the total effective

TABLE 1: CLINICAL DATA

\begin{tabular}{|c|c|c|c|c|c|c|c|}
\hline \multirow{2}{*}{ Group } & \multirow{2}{*}{$\mathrm{n}$} & \multirow{2}{*}{$\begin{array}{c}\text { Average age } \\
\text { (y) }\end{array}$} & \multirow{2}{*}{ BMI } & \multicolumn{2}{|c|}{ Cancer measurement } & \multicolumn{2}{|c|}{ Marital status } \\
\hline & & & & Left & Right & Married & Single \\
\hline Group NH & 68 & $45.23 \pm 3.03$ & $26.23 \pm 3.45$ & 28 & 32 & 50 & 8 \\
\hline Group CG & 68 & $45.31 \pm 2.87$ & $26.19 \pm 3.71$ & 27 & 31 & 49 & 9 \\
\hline$\chi^{2 / t}$ & & 0.158 & 0.065 & \multicolumn{4}{|c|}{0.069} \\
\hline$P$ & & 0.874 & 0.948 & \multicolumn{4}{|c|}{0.702} \\
\hline
\end{tabular}


rate of CG group was significantly lower $(\mathrm{p}<0.05)$ as shown in Table 2. There was no significant difference in the levels of plasma E1, E2 and FSH between the $\mathrm{NH}$ group and $\mathrm{CG}$ group before treatment $(\mathrm{p}<0.05)$. Compared with the $\mathrm{NH}$ group, the plasma levels of E1 and E2 in CG group was significantly higher after treatment, while compared to the CG group, the level of serum FSH in NH group was significantly higher after treatment $(p<0.05)$ as shown in Table 3. There was no significant difference in tumor size between the $\mathrm{NH}$ group and CG group before treatment $(\mathrm{p}>0.05)$. After treatment, the tumor volume of each group gradually decreased with increasing treatment time, and the tumor reduction degree of CG group was lower than that of NH group $(p<0.05)$ as shown in Table 4 . The number of leucopenia cases in the NH group was 14 , and that in the CG group were 17, slightly more than that in the NH group, but not significantly higher as shown in Table 5. After 2 y of follow-up, the survival rate of NG group was significantly higher than that of CG group $(p<0.05)$ as shown in fig. 1 .

Over the years, breast cancer showed a high mortality rate. Every year, more than 200000 women are diagnosed with breast cancer all over the world. The causes of breast cancer are complex and involve many factors. However, at present, there are very limited surgical and therapeutic interventions available for treating breast cancer. In addition, chemotherapy drugs in clinical use currently have certain toxicity and lead to many side effects. At the same time drug resistance towards chemotherapy drugs also appear in patients with adenocarcinoma ${ }^{[9,10]}$. Similar to the occurrence of other tumors, breast cancer development is a longterm and multi-step process, involving complex genetic and epigenetic changes ${ }^{[11]}$. Although in the past few decades, many studies have explored the pathogenesis of breast cancer, at present, the molecular mechanism of the occurrence and development of breast cancer is

TABLE 2: CLINICAL EFFICACY OF THE NH AND CG GROUPS

\begin{tabular}{lccccc}
\hline Group & $\mathbf{n}$ & Complete remission & Partial remission & Disease stabilization & Total effective rate \\
\hline Group Nh & 68 & $9(13.2 \%)$ & $35(51.4 \%)$ & $2(33.8 \%)$ & $44(64.7 \%)$ \\
Group Cg & 68 & $4(5.8 \%)$ & $12(17.6)$ & $39(57.3 \%)$ & $16(23.5 \%)$ \\
$X^{2}$ & & & & 15.562 \\
P & & & & 0.002 \\
\hline
\end{tabular}

TABLE 3: CHANGES IN SERUM E1, E2 AND FSH IN NH AND CG GROUPS

\begin{tabular}{lccccccc}
\hline & \multirow{2}{*}{ Group } & $\mathrm{n}$ & $\begin{array}{c}\text { Before } \\
\text { treatment }\end{array}$ & After treatment & $\begin{array}{c}\text { Before } \\
\text { treatment }\end{array}$ & After treatment & \multicolumn{2}{c}{ Before } \\
treatment & After treatment \\
\hline Group NH & 68 & $68.19 \pm 5.2$ & $35.46 \pm 3.35^{\#}$ & $82.65 \pm 5.61$ & $53.91 \pm 4.72^{\#}$ & $50.23 \pm 5.09$ & $74.56 \pm 7.15^{\#}$ \\
Group CG & 68 & $68.23 \pm 5.12$ & $50.34 \pm 4.62^{\#}$ & $82.71 \pm 5.48$ & $63.03 \pm 6.28 \#$ & $50.36 \pm 4.78$ & $65.23 \pm 6.22^{\#}$ \\
$\mathrm{t}$ & & 0.038 & 18.44 & 0.054 & 8.209 & 0.131 & 6.961 \\
$\mathrm{P}$ & 0.969 & $<0.001$ & 0.956 & $<0.001$ & 0.895 & $<0.001$ \\
\hline
\end{tabular}

"Means comparison with the same group before treatment $(\mathrm{p}<0.05)$

TABLE 4: TUMOR SIZE AFTER 1-3 COURSES OF TREATMENT IN THE NH AND CG GROUPS

\begin{tabular}{lcccccc}
\hline Group & $\mathrm{n}$ & Before treatment & First course of treatment & Second course of treatment & $\mathrm{F}$ & $\mathrm{P}$ \\
\hline Group Nh & 68 & $47.25 \pm 13.14$ & $36.23 \pm 10.21^{*}$ & $19.75 \pm 12.03^{* *}$ & 77.63 & $<0.001$ \\
Group Cg & 68 & $47.30 \pm 12.36$ & $41.15 \pm 10.36^{*}$ & $35.26 \pm 11.58^{* \#}$ & 7.894 & $<0.001$ \\
$\mathrm{t}$ & & 0.019 & 2.392 & 6.568 & & \\
$\mathrm{P}$ & & 0.984 & 0.018 & $<0.001$ & & \\
\hline
\end{tabular}

*Means comparison with the pretreatment $(\mathrm{p}<0.05)$, \#means comparison with the first course $(\mathrm{P}<0.05)$, "means comparison with the second course $(p<0.05)$

TABLE 5: ADVERSE REACTIONS OF THE NH AND CG GROUPS

\begin{tabular}{lcccccc}
\hline Group & $\mathbf{n}$ & $\begin{array}{c}\text { Nausea and } \\
\text { vomiting }\end{array}$ & Alopecia & Leukopenia & Central granulocytopenia & $\begin{array}{c}\text { Peripheral } \\
\text { neurotoxicity }\end{array}$ \\
\hline Group Nh & 68 & 12 & 12 & 14 & 11 & 13 \\
Group Cg & 68 & 17 & 15 & 17 & 18 & 15 \\
$\mathrm{X}^{2}$ & & 0.711 & 0.278 & 0.237 & 1.395 & 0.118 \\
$\mathrm{P}$ & & 0.399 & 0.598 & 0.626 & 0.237 & 0.731 \\
\hline
\end{tabular}




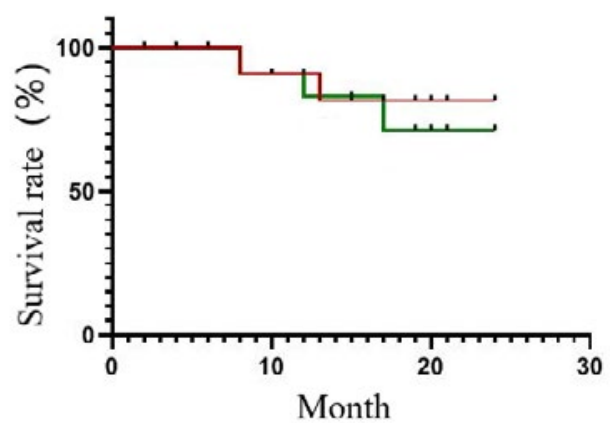

Fig. 1: Two-year survival rate of NH group and CG group (一) Group NH, (-) group CG

still poorly understood. The clinical outcome of patients after surgery is poor, and the long-term prognosis is not ideal $^{[12]}$.

In this study, it was found that in the $\mathrm{NH}$ group there were 9 complete remission cases, 35 partial remission cases, 23 disease stable cases while, 8 cases showed disease progression. On the other hand in the CG group, only 4 complete remission cases, 12 partial remission cases, 39 disease stable cases and 13 disease progression cases were seen. The total effective rate of $\mathrm{NH}$ group was higher than that of CG group. Compared with the NH group, the serum E1 and E2 levels of the CG group were significantly higher, and compared with the CG group, the serum FSH levels of the $\mathrm{NH}$ group were significantly higher. It was reported that many hormones such as E1, E2, FSH in the female can promote the proliferation and invasion of cancer cells and the mechanism may be related to the activation of proteins, enzymes or oncogenes involved in nucleic acid synthesis induced by these hormones, so as to increase the growth of cancer cells ${ }^{[13,14]}$. Conventional macromolecular chemotherapy drugs are difficult to be absorbed. Low concentration chemotherapy drugs have little effect on cancer cells. Epirubicin, an anthracycline drug, would show a higher concentration in the tumor, which could kill the cells before these invade other organs, fundamentally reducing the possibility of recurrence and metastasis of cancer ${ }^{[15,16]}$. Some studies have confirmed that epirubicin has outstanding ability to penetrate into cancer cells. After embedding into DNA base pairs, it can closely combine with DNA resulting in the reduction of DNA replication leading to reduction in DNA-dependent polymerase activity and RNA transcription, thus inhibiting tumor growth with $\mathrm{in}^{[17]}$, which is consistent with the results obtained in this investigation.

In this study, it was found that the number of neutropenia cases in the NH group was 14 at most, and the number of neutropenia cases in the CG group was 17, and the number of adverse reactions in the $\mathrm{CG}$ group was significantly higher than that in the $\mathrm{NH}$ group. The survival rate of the patients in the $\mathrm{NH}$ group was significantly higher than that in the CG group. After treatment, the tumor volume in each group decreased gradually with increasing treatment time and the tumor reduction degree of $\mathrm{CG}$ group was lower than that of $\mathrm{NH}$ group. According to reported literature, chemotherapy could inhibit tumor neovascularization and different drugs have different effects, but could induce tumor cell apoptosis. After treatment, tumor volume could be significantly reduced ${ }^{[18]}$. The clinical outcome of single use or combined use is considerable and the incidence of cardiotoxicity of epirubicin is significantly lower than that of Adriamycin ${ }^{[19]}$. Some studies have confirmed that the combination of epirubicin with other drugs has a significant effect in the treatment of breast cancer. Compared to conventional chemotherapy drugs, the adverse reactions are few and the prognosis is better, which is worth promoting its clinical use ${ }^{[20]}$. To sum up, anthracycline is effective in the treatment of advanced breast cancer, the level of serum E2 is decreased and the tumor volume is reduced.

\section{Conflict of interest:}

Authors reported no conflicts of interest.

\section{REFERENCES}

1. Hoppe R, Fan P, Winter S, Büttner F, Jordan VC, Brauch HB. Abstract 474: Estrogen-responsive miRNAs as modulators of E2-induced apoptosis in AI-resistant breast cancer. J Can Res 2017;77:474-4.

2. Bastiaannet E, van de Water W, Liefers GJ, van de Velde CJ. E2. Controversial issues in the surgical management of the elderly breast cancer patient. Euro J Cancer 2012;48:S4-S5.

3. Huang LY. Study on the effect of taxanes and anthracyclines on the short-term efficacy of breast cancer patients. Chin J Pract Med 2016;43:56-8.

4. Shen S, Xu Y, Zhou Y, Mao F, Guan J, Sun Q. Concurrent administration of trastuzumab and anthracyclines as adjuvant regimen for HER2-positive breast cancer: a randomized controlled trial. Oncotarget 2017;8:92778-87.

5. $\mathrm{Hu}$ GF, Fu HX, Ma JF. Clinical study on the effect of anthracycline drugs on cardiac toxicity in patients with early breast cancer. Chin J Cardiovasc Dis 2018;46:987-92.

6. Zhang MM, Luo F. Cardiovascular risk and strategy of anthracycline in the treatment of breast cancer. Cancer Res Clin, 2018;30:173-5.

7. Ligibel JA, Winer EP. Trastuzumab/chemotherapy combinations in metastatic breast cancer. Semin Oncol 2002;29:38-43.

8. Yndestad S, Austreid E, Svanberg IR, Knappskog S, Lønning PE, Eikesdal HP. Activation of Akt characterizes estrogen receptor positive human breast cancers which respond to anthracyclines. Oncotarget 2017;8:41227-241.

9. $\mathrm{Hu}$ YZ. The effect of different combinations of trastuzumab 
and anthracycline on breast cancer and heart function. J Pract Cancer 2016;31:1684-6.

10. Chen FL, Wang K. The relationship between the TOP2A gene of breast cancer and the efficacy and prognosis of anthracycline drugs. Evid Based Med 2016;16:247-52.

11. Shee K, Kono AT, D'Anna SP, Seltzer MA, Lu X, Miller TW, et al. Maximizing the Benefit-Cost Ratio of Anthracyclines in Metastatic Breast Cancer: Case Report of a Patient with a Complete Response to High-Dose Doxorubicin. Case Rep Oncol 2016;9:840-46.

12. Liu W, Li JB, Wang T. Evaluation of the efficacy and safety of anthracycline combined with Taxus in neoadjuvant chemotherapy for breast cancer. J Clin Oncol 2016;21:228-32.

13. Fernando A, Jayarajah U, Prabashani S, Fernando EA, Seneviratne SA. Incidence trends and patterns of breast cancer in Sri Lanka: An analysis of the national cancer database. BMC Cancer 2018;18:482.

14. Caughran J, Braun TM, Breslin TM, Smith DR, Kreinbrink JL, Parish GK, et al. The Effect of the 2009 USPSTF breast cancer screening recommendations on breast cancer in Michigan: A longitudinal study. Breast J 2018;24:730-37.

15. Chen DB, Chen B, Yang HJ. Study on the effect of anthracyclines on ER, VEGF-C, VEGFR-3 and E-cadherin in serum of breast cancer patients. Chin J Biochem Med 2015;35:137-9.

16. Zhou N, Zeng HQ, Liu SH. Correlation between tumor volume change and HER2 expression in neoadjuvant chemotherapy of breast cancer by ABVS. Chin J Ultrasound Med 2018;34: 413-7.

17. Vetter M, Fokas S, Biskup E, Schmid T, Schwab F, Schoetzau A, et al. Efficacy of adjuvant chemotherapy with carboplatin for early triple negative breast cancer: a single center experience. Oncotarget 2017;8:75617-26.

18. He X. Clinical effect of neoadjuvant chemotherapy with epirubicin and paclitaxel in triple negative breast cancer patients. Oncol Lett 2017;15:60-2.

19. Aleck K, Hallman K, Quigley M, Lloyd V, Szmyd M, Ruskin $\mathrm{D}$, et al. Effects of Atrial Natriuretic Peptide on p53 and Estrogen Receptor in Breast Cancer Cells. Bioresearch Open Access 2017;6:141.

20. Smirnova T, Bonapace L, MacDonald G, Kondo S, Wyckoff $\mathrm{J}$, Ebersbach $\mathrm{H}$, et al. Serpin E2 promotes breast cancer metastasis by remodeling the tumor matrix and polarizing tumor associated macrophages. Oncotarget 2016;7:82289.

This is an open access article distributed under the terms of the Creative Commons Attribution-NonCommercial-ShareAlike 3.0 License, which allows others to remix, tweak, and build upon the work non-commercially, as long as the author is credited and the new creations are licensed under the identical terms

This article was originally published in a special issue, "Recent Trends in Biomedical Research" Indian J Pharm Sci 2020:82(1)spl issue1; XX-XX 